Canadian Science Publishing

Applied Physiology, Nutrition, and Metabolism Physiologie appliquée, nutrition et métabolisme

\title{
Influence of a low carbohydrate diet on endothelial microvesicles in overweight women
}

\begin{tabular}{|c|c|}
\hline Journal: & Applied Physiology, Nutrition, and Metabolism \\
\hline Manuscript ID & apnm-2015-0507.R1 \\
\hline Manuscript Type: & Article \\
\hline Date Submitted by the Author: & 01-Dec-2015 \\
\hline Complete List of Authors: & $\begin{array}{l}\text { Wekesa, Antony; Waterford Institute of Technology, Schools of Health } \\
\text { Science and Science and Computing } \\
\text { Doyle, Lorna; Waterford Institute of Technology, Department of Health, } \\
\text { Sport and Exercise Science } \\
\text { Fitzmaurice, Doreen; Waterford Institute of Technology, Department of } \\
\text { Health, Sport and Exercise Science } \\
\text { O'Donovan, Orla; Waterford Institute of Technology, School of Science and } \\
\text { Computing } \\
\text { Phelan, John; Waterford Institute of Technology, School of Science and } \\
\text { Computing } \\
\text { Ross, Mark; Edinburgh Napier University; Waterford Institute of } \\
\text { Technology, Department of Health, Sport and Exercise Science } \\
\text { Cross, Keith; University Hospital Waterford, Department of Vascular } \\
\text { Surgery; Royal College of Surgeons in Ireland } \\
\text { Harrison, Michael; Waterford Institute of Technology, Schools of Health } \\
\text { Science and Science and Computing }\end{array}$ \\
\hline Keyword: & vascular health, endothelial biomarkers, weight loss, microparticles \\
\hline
\end{tabular}

\section{SCHOLARONE"}

Manuscripts 


\section{Title page}

\section{Title}

Influence of a low carbohydrate diet on endothelial microvesicles in overweight women

\section{Running head}

Low carb diets and endothelial microvesicles

\section{Authors}

Antony L. Wekesa ${ }^{1}$, Lorna M. Doyle ${ }^{1}$, Doreen Fitzmaurice ${ }^{1}$, Orla O’Donovan ${ }^{1}$, John P.

Phelan $^{1}$, Mark D. Ross ${ }^{1,2}$, Keith S. Cross ${ }^{3,4}$, Michael Harrison ${ }^{1}$

${ }^{1}$ Schools of Health Science and Science and Computing, Waterford Institute of

Technology, Waterford, Ireland

${ }^{2}$ School of Life, Sport and Social Science, Edinburgh Napier University, Edinburgh,

United Kingdom

${ }^{3}$ University Hospital Waterford, Waterford, Ireland

${ }^{4}$ Royal College of Surgeons in Ireland, Dublin, Ireland

\section{Corresponding author}

Michael Harrison

School of Science and Computing,

Waterford Institute of Technology,

Waterford,

Ireland.

Email: mharrison@wit.ie

Tel: +353-51-302027 


\begin{abstract}
Low carbohydrate diets (LCD) are increasing in popularity, but their effect on vascular health has been questioned. Endothelial microvesicles (EMV) are membrane-derived vesicles with the potential to act as a sensitive prognostic biomarker of vascular health and endothelial function. The aim of this study was to examine the influence of a LCD on EMV and other endothelial biomarkers of protein origin. Twenty-four overweight women (aged $48.4 \pm 0.6 \mathrm{y}$, height $1.60 \pm 0.07 \mathrm{~m}$, body mass $76.5 \pm 9.1 \mathrm{~kg}$, BMI $28.1 \pm 2.7$ $\mathrm{kg} \cdot \mathrm{m}^{-2}$, waist circumference $84.1 \pm 7.4 \mathrm{~cm} ;$ mean $\pm \mathrm{SD}$ ) were randomised to either 24 weeks on their normal diet (ND) or a LCD after which they crossed over to 24 weeks on the alternative diet. Participants were assisted in reducing carbohydrate intake but not below $40 \mathrm{~g} \mathrm{day}^{-1}$. Body composition and endothelial biomarkers were assessed at the cross-over point and at the end of the study. Daily carbohydrate intake ( $87 \pm 7 \mathrm{vs.} 179 \pm 11 \mathrm{~g})$ and the percentage of energy derived from carbohydrate $(29$ vs. $44 \%)$ were lower $(p<0.05)$ on the LCD compared to the ND but absolute fat and saturated fat intake were unchanged. Body mass and waist circumference were $3.7 \pm 0.8 \mathrm{~kg}$ and $3.5 \pm 1.0 \mathrm{~cm}$ lower $(\mathrm{p}<0.05)$ respectively after the LCD compared with the ND phases. $\mathrm{CD} 31^{+} \mathrm{CD} 41^{-} \mathrm{EMV}$, soluble (s) thrombomodulin, sE-selectin, sP-selectin, serum amyloid A and C-reactive protein were lower $(\mathrm{p}<0.05)$ after the LCD compared to the ND, but serum lipids and apolipoproteins were not different. EMV along with a range of endothelial and inflammatory biomarkers are reduced by a LCD that involves modest weight loss.
\end{abstract}

KEYWORDS: vascular health; endothelial biomarkers; weight loss; microparticles 


\section{Introduction}

Low carbohydrate diets (LCD) are increasing in popularity as a means of achieving weight loss in overweight women. LCD typically have a carbohydrate content of less than $100 \mathrm{~g} d a y^{-1}$ or less than $30 \%$ of total daily energy intake (Bilsborough \& Crowe, 2003). Diets such as the Atkins diet, sometimes termed very low carbohydrate diets or very low carbohydrate ketogenic diets, target a carbohydrate content of $20-50 \mathrm{~g}^{\cdot d a y}{ }^{-1}$, representing less than $20 \%$ of total energy intake. LCD generally show greater weight loss at 6 mo when compared to low fat diets (Brehm et al., 2003; Foster et al., 2003; Gardner et al., 2007; Samaha et al., 2003; Yancy et al., 2004). The results of a metaanalysis of early trials demonstrate $3.3 \mathrm{~kg}$ greater weight loss at 6 mo when LCD are compared to low fat calorie-restricted diets and at least as much weight loss at 12 mo (Nordmann et al., 2006). Despite these results with respect to body weight, the safety of LCD have been questioned (Bilsborough \& Crowe, 2003; Frigolet et al., 2011). A reduction in body mass of 5-10\% following dietary restriction has the potential to improve vascular risk profile (National Institutes of Health, 1998). However, individuals on LCD typically obtain a greater proportion of energy from fat, saturated fat and protein which may have pro-atherogenic effects on some risk markers. In their meta-analysis, Nordman and colleagues (Nordmann et al., 2006) demonstrated pro-atherogenic changes in serum total cholesterol and low density lipoprotein cholesterol (LDL-C) following LCD compared to low fat diets but anti-atherogenic changes in serum triglycerides, high density lipoprotein cholesterol (HDL-C) and blood pressure. Given these conflicting findings, there is a need to monitor more than traditional risk factors when assessing the impact of dietary interventions on vascular risk. 
The endothelium expresses and releases a wide variety of factors relevant to vascular inflammation, coagulation, vessel tone and barrier function. Endothelial-derived factors associated with the atherothrombotic process have the ability to monitor changes in vascular risk following lifestyle interventions. Soluble forms of selectins and cell adhesion molecules have been employed previously in intervention studies (Davis et al., 2011; Keogh et al., 2008; Porreca et al., 2004; Sharman \& Volek, 2004; Wycherley et al., 2009). Selectins and adhesion molecules are involved in the rolling and tethering of circulating leukoctyes and their migration into the subendothelial layer, key processes in atherogenesis (Brevetti et al., 2006). Thrombomodulin is a key component of endothelial thromboresistance with cell surface expression reduced, and circulating levels increased, with endothelial injury and dysfunction (Martin et al., 2013).

Microvesicles are a novel class of vascular biomarker released from a range of vascular cells including endothelial cells, platelets, vascular smooth muscle cells, erythrocytes and leukocytes, into a range of body fluids including blood, urine, cerebrospinal fluid, lymph, saliva and tears. These small vesicles, between $100-1000 \mathrm{~nm}$ in diameter, are released by outward budding of the parent cell plasma membrane. In contrast, exosomes, a related class of extracellular vesicle between $30-100 \mathrm{~nm}$ in diameter, are released by exocytosis. Microvesicles are not simply biomarkers but also potentially mediators of disease activity and cell-cell communication, transporting bioactive lipids, membrane proteins, mRNA and miRNA from the parent to target cells (Akers et al., 2013; Andriantsitohaina et al., 2012; Ayers et al., 2015). Endothelial microvesicles (EMV) 
have prognostic potential as vascular biomarkers that may exceed the more established soluble protein biomarkers. They are elevated in a range of vascular inflammatory disorders and add to risk prediction in prospective studies when added to models involving more established markers (Nozaki et al., 2009). Recently, we have shown an association between EMV and unstable atherosclerosis (Wekesa et al., 2014). EMV impair endothelial dependent dilation, increase arterial stiffness, promote inflammation and initiate coagulation (Chironi et al., 2009).

In contrast to studies that have assessed blood lipids, blood glucose and blood pressure, only a few studies have employed endothelial biomarkers to assess the effects of LCD on vascular risk, and to our knowledge no studies have employed EMV. Reductions in soluble intercellular adhesion molecule (sICAM-1), soluble E-selectin and soluble Pselectin, (Davis et al., 2011; Keogh et al., 2008; Sharman \& Volek, 2004; Wycherley et al., 2009) been demonstrated following LCD interventions, but not soluble vascular cell adhesion molecule (sVCAM-1) (Wycherley et al., 2009). Although elevated in the obese state (Stepanian et al., 2013), EMV have not been widely employed in dietary intervention research. Following bariatric surgery-induced weight loss however, one study reported a reduction in EMV (Cheng et al., 2013) and another no change (Stepanian et al., 2013).

The purpose of this study was to examine the influence of a 24-week LCD on cardiovascular risk profile determined from changes in EMV and other endothelial- 
inflammatory biomarkers. Middle-aged overweight women were chosen as the subject group of interest, given the popularity of LCD and the paucity of data in this population.

\section{Methods}

Study overview

In this randomised crossover study, participants were randomised to either 24 weeks on their normal diet (ND) or a LCD after which they crossed over to the alternative diet for a further 24 weeks. A fasting blood sample was obtained at the cross-over point (Week 24) and at the end of the study (Week 48) from which circulating microvesicles and protein biomarkers were determined. Body composition and blood pressure were also evaluated at these time points.

\section{Study population and ethics}

Twenty-eight women were recruited into this intervention study though 4 dropped out at various stages. The final analysis was therefore based on 24 participants (aged $48.4 \pm 0.6$ $\mathrm{y}$, height $1.60 \pm 0.07 \mathrm{~m}$, body mass $76.5 \pm 9.1 \mathrm{~kg}$, body mass index (BMI) $28.1 \pm 2.7$ $\mathrm{kg}^{-2}$, waist circumference $84.1 \pm 7.4 \mathrm{~cm}, \%$ body fat $43.5 \pm 5.0$; mean $\pm \mathrm{SD}$ ) who completed both phases of the study. The inclusion criteria were women in their late reproductive or post-menopausal years, a BMI between 25 and $30 \mathrm{kgm}^{-2}$ and not having engaged in dieting practices in the previous 6 months. Exclusion criteria included participation in intense physical activity ( $>3$ times per week of vigorous physical activity), high alcohol consumption ( $>14$ units weekly), currently pregnant, lactating or taking hormone replacement therapy, previous hysterectomy or a history of chronic 
menstrual irregularities, diabetes mellitus, kidney disease, chronic illness, inflammatory conditions, renal, gastrointestinal or hormonal disorders. Each participant completed a health screening form to determine eligibility for the study. From an ethical standpoint, the study was conducted in accordance with the Declaration of Helsinki. Ethical approval was obtained from Waterford Institute of Technology Research Ethics Committee and written informed consent was obtained from each participant. Details of the trial protocol have been published (www.isrctn.com, ISRCTN 48034106).

\section{Diet}

During the LCD period participants were assisted in to reducing their carbohydrate intake considerably, but not below $40 \mathrm{gday}^{-1}$. Participants were also provided with information on what constituted carbohydrate foods, a list of the macronutrient content in the most commonly consumed foods and a booklet with LCD recipes, meal plans and advice. In addition, they attended cooking classes that demonstrated how to make LCD meals that were not readily available in stores. Participants completed weekly food frequency questionnaires for self-monitoring purposes and to assist with compliance. These were discussed with participants at the mid-point of each phase but were not analysed for research purposes. Four individuals exited the study because of an inability to adhere to a LCD. In contrast, the ND group were monitored via the weekly food frequency questionnaires.

\section{Dietary assessment}

A 3 day food diary was completed at baseline and at weeks 12 (mid-point phase 1), 24 (cross over), 36 (mid-point phase 2) and 48 (end of study). Participants were instructed to maintain current phase-dependent dietary practices and to estimate food quantities. 
Visual and instructional cues were given to assist with portion size estimation. The food diaries were analysed using CompEat ${ }^{\mathrm{TM}}$ analysis software. Intake of selected macronutrients (energy, carbohydrate, fat, saturated fat and protein) and micro-nutrients during the ND and LCD phases were calculated by averaging daily values at the mid-point and end of each phase from these 3 day food diary analyses.

\section{Physiological Measurements}

Height, body mass, waist circumference and blood pressure were measured at baseline, week 24 and week 48. Blood pressure was determined using an automated blood pressure monitor (Omron Healthcare, USA) and appropriate cuff size, with participants seated and resting for $10 \mathrm{~min}$ before assessments were made. Body fat was determined by DEXA (Norland XR-46, USA).

\section{Blood collection and processing}

All blood samples were taken in the morning following an overnight fast. The first 3.0 $\mathrm{mL}$ was discarded. Samples were kept at room temperature prior to centrifugation and processed within 2 hours of collection. The serum tubes were centrifuged for 15 minutes at $1500 \mathrm{~g}$ at $20^{\circ} \mathrm{C}$. The upper part of the serum and plasma was collected down to $1 \mathrm{~cm}$ above the buffy coat layer. The sodium citrate tubes (3.2\%), for MV analysis, underwent a double centrifugation protocol. They were first centrifuged for 15 minutes at $1500 \mathrm{~g}$ at $20^{\circ} \mathrm{C}$, followed by 2 minutes at $13,000 \mathrm{~g}$ at $20^{\circ} \mathrm{C}$ to produce platelet poor plasma, with the pellet discarded. Aliquots of $\sim 350 \mathrm{~mL}$ of serum and plasma were stored at $-80^{\circ} \mathrm{C}$ for later analysis. 


\section{Microvesicle and soluble protein biomarker analysis}

MV were defined based on size and fluorescence using flow cytometry (FC500, Beckman Coulter, USA). Microvesicles were defined as particles $<1.0 \mu \mathrm{m}$ in diameter with fluorescence greater than that of negative control samples. A microvesicle size gate was established on forward vs. side scatter using 0.5 and $0.9 \mu \mathrm{m}$ sizing beads (Megamix, Biocytex, France). This bead combination can be used to establish the upper limit of the size gate and to standardize MV enumeration by calibrating the lower limits of the gate just above the noise of the instrument. Two different EMV subsets were enumerated, MV expressing CD31 but not expressing the platelet marker CD41 (CD31 $\left.{ }^{+} \mathrm{CD}^{-} 1^{-} \mathrm{EMV}\right)$ and MV expressing the endothelial specific markers CD144, CD146 or CD105 in a monochrome multimarker assay (mmEMV). This monochrome multimarker approach was previously employed (Duval et al., 2010) to improve the signal to noise ratio, given the low number of these endothelial specific markers on particles of small surface area. Representative plots are included as supplementary data (supplementary figure S1).

Plasma samples were thawed on ice prior to analysis. Exactly $330 \mu \mathrm{l}$ of thawed plasma was washed twice in PBS-citrate $(19,000 \mathrm{~g}$ for $30 \mathrm{~min})$. After the first wash the pellet ( $30 \mu \mathrm{L}$ ) was resuspended in PBS-citrate. Following the second wash the pellet was resuspended in $80 \mu \mathrm{L}$ PBS-citrate and vortexed thoroughly, resulting in a sufficient sample volume for all assays (CD3 $1^{+} 41^{-}$, mmEMV and negative control samples). The following reagents were used to stain the samples, CD31-PE, CD41-PECy5, CD144-PE, CD146-PE, CD105-PE, IgG1-PECy5 and IgG1-PE (all BD Biosciences, UK). All antibodies were diluted in PBS and centrifuged at $13,000 \mathrm{~g}$ for 3 min to remove 
aggregates. For the $\mathrm{CD} 31^{+} 41^{-}$EMV assay, $20 \mu \mathrm{L}$ of resuspended pellet was incubated in the dark for $30 \mathrm{~min}$ with CD31-PE (final conc. $0.68 \mu \mathrm{g} \cdot \mathrm{mL}^{-1}$ ) and CD41-PECy5 (final conc. $0.12 \mu \mathrm{gmL}^{-1}$ ). The negative control sample employed IgG1-PECy5. For the mmEMV assay, $20 \mu \mathrm{L}$ of resuspended pellet was incubated in the dark for 30 min with CD144-PE (final conc. $0.19 \mu \mathrm{g}^{\mathrm{m}} \mathrm{mL}^{-1}, \mathrm{CD} 146-\mathrm{PE}$ (final conc. $0.12 \mu \mathrm{g} \cdot \mathrm{mL}^{-1}$ ) and CD105PE (final conc. $0.38 \mu \mathrm{gmL}^{-1}$ ). The negative control employed IgG1-PE (final conc. 0.69 $\left.\mu \mathrm{gmL}^{-1}\right)$. Then, $500 \mu \mathrm{L}$ of phosphate buffered saline was added to each sample, followed immediately by flow cytometric analysis for $3 \mathrm{~min}$ at medium speed. Instrument flow rate was calculated using flow count beads (SPHERO Accucount, Spherotech, USA). EMV counts were converted to event $\mu 1^{-1}$ taking into account the flow rate of the instrument, the $500 \mu \mathrm{l}$ of PBS added prior to analysis and the concentrating effects of the wash protocol which reduced a starting plasma volume from $330 \mu 1$ to $110 \mu 1$.

Endothelial-inflammatory biomarkers of protein origin were analysed in duplicate using commercially available immunoassays (Meso Scale Discovery, Rockville, MD, USA) employing multiplexing technology and electrochemiluminescence detection. Serum cholesterol, high density lipoprotein (HDL) cholesterol, low density lipoprotein (LDL) cholesterol, triglycerides, apolipoprotein A1 (ApoA1), and apolipoprotein B (ApoB) were determined via spectrophotometric assays performed on an automated clinical chemistry system (ACE® Wassermann B.V., Netherlands) using appropriate reagents, calibrators and controls (Randox Laboratories, UK). 


\section{Statistics}

All data was checked for normality using the Kolmogorov-Smirnov test. The effect of the LCD on all variables was determined by comparing values at the end of the ND and LCD phases using paired t-tests. The potential for carryover between phase 1 and phase 2 of the study was checked by comparing the delta scores (ND - LCD) for each variable between the group that was first randomised to the ND phase $(n=12)$ and the group first randomised to the LCD phase $(n=12)$ using independent t-tests (Wellek \& Blettner, 2012). Associations between the between-phase differences in body mass and the differences in circulating biomarkers were determined using Pearson correlations. Significance was set at $\mathrm{p}<0.05$. Data are presented as mean $\pm \mathrm{SEM}$.

\section{Results}

\section{Carryover}

With respect to body mass, blood pressure, EMV and all soluble endothelial and inflammatory biomarkers, delta scores between the ND and LCD phases were not different between the group first randomised to the ND phase and the group first randomised to the LCD phase.

\section{Mean daily intake of various nutrients}

Daily intake of energy and macronutrients on the ND and LCD (table 1) were calculated by averaging the 3 day food diary analysis at the mid-point and end of each phase. Mean daily energy and carbohydrate intake were $398 \mathrm{kcal}$ and $92 \mathrm{~g}$ lower $(\mathrm{p}<0.05)$ respectively on the LCD compared to the ND. Mean daily intake of fat, saturated fat and protein was 
not different on the LCD and ND (table 1) but the percentage of energy obtained from fat and protein was higher $(\mathrm{p}<0.05)$ on the LCD compared to the ND (figure 1$)$.

\section{Body composition}

Body mass, body mass index and waist circumference were $3.7 \pm 0.8 \mathrm{~kg}, 2.4 \pm 0.3 \mathrm{kgm}^{-2}$ and $3.5 \pm 1.0 \mathrm{~cm}$ lower respectively $(\mathrm{p}<0.05)$ at the end of the LCD compared to the ND phases (table 2). Systolic blood pressure was not different but diastolic blood pressure was $5 \pm 2 \mathrm{mmHg}$ lower at the end of the LCD compared to the ND phases.

EMV and endothelial-inflammatory biomarkers

$\mathrm{CD} 31^{+} \mathrm{CD} 41^{-}$EMV $(\mathrm{p}<0.05)$ but not $\operatorname{mmEMV}(\mathrm{p}=0.08)$ were lower at the end of the LCD compared to the end of the ND (table 3). Soluble E-selectin, sP-selectin, soluble thrombomodulin (sTM), C-reactive protein (CRP) and serum amyloid A (SAA) were lower $(\mathrm{p}<0.05$ ) following 24 weeks on the LCD compared to 24 weeks on the ND (table 3). Soluble ICAM-1, sICAM-3 ( $p=0.08)$ and sVCAM-1 were not different following 24 weeks on the LCD compared to the ND (table 3). Serum lipids, apolipoproteins and glucose were not different at the end of the LCD compared to the ND phases (table 4).

\section{Associations}

The difference in $\mathrm{CD} 31^{+} \mathrm{CD} 41^{-} \mathrm{EMV}$ between dietary phases was correlated with the difference in mmEMV $(\mathrm{r}=0.51)$ but with no other biomarker or dietary variable. The difference in waist circumference was correlated $(\mathrm{p}<0.05)$ with the difference in mmEMV $(\mathrm{r}=0.47)$. The difference in body mass between phases was correlated $(\mathrm{p}<0.05)$ with the difference in diastolic blood pressure $(\mathrm{r}=0.44)$, sE-selectin $(\mathrm{r}=0.68)$ and CRP $(\mathrm{r}=0.56)$. 


\section{Discussion}

The purpose of this study was to determine the influence of a 24 week LCD on EMV and soluble endothelial biomarkers of protein origin in overweight women. CD $31^{+} 41^{-} \mathrm{EMV}$, sE-selectin, sP-selectin, sTM, SAA and CRP were all reduced as a result of the LCD. These changes were accompanied by a modest reduction in body mass, waist circumference and diastolic blood pressure.

This dietary intervention targeted a reduction in carbohydrate intake but not the near elimination of carbohydrate from the diet. Participants were not permitted to reduce carbohydrate intake below $40 \mathrm{~g} d a y^{-1}$ in order to avoid ketogenesis. Otherwise the participants were free to devise their own strategies to help them achieve the reduction in carbohydrate intake. Carbohydrate intake was $\sim 90 \mathrm{~g}^{\cdot d a y}{ }^{-1}$ lower on the LCD compared to the ND. With no meaningful increase in absolute fat or protein intake when consuming the LCD, the resultant daily energy deficit was $\sim 400 \mathrm{kcal}$. However, the percentage of energy obtained from carbohydrate decreased from $44 \%$ to $28 \%$ with the percentage of energy from fat increasing from $38 \%$ to $47 \%$ and protein from $17 \%$ to $23 \%$. There was no difference in saturated fat intake between the LCD and ND phases, whether expressed in gday $^{-1}$ or as a $\%$ of total fat intake. The energy deficit over the course of the 24 weeks resulted in a moderate reduction in body mass and waist circumference, respectively. The percentage reduction in body mass was $\sim 5 \%$, which according to some sources (National Institutes of Health, 1998) is the threshold at which health benefits are observed. 
This is the first study to demonstrate a reduction in EMV and adds to the body of evidence demonstrating an improvement in cardiovascular risk profile following a reduction in dietary carbohydrate intake leading to moderate weight loss. EMV are emerging as a novel marker of vascular risk in cross-sectional but also in prospective studies (Lee et al., 2012; Nozaki et al., 2009) where they can add to risk prediction. This reduction in $\mathrm{CD} 31^{+} \mathrm{CD} 41^{-} \mathrm{EMV}$ was accompanied by a reduction in soluble endothelial markers and acute phase inflammatory proteins. The difference in the mmEMV subset between diet phases did not reach statistical significance. There is evidence that specific EMV subsets exist and respond differently to cell activation and apoptosis (Jimenez et al., 2003). Two other studies have examined changes in EMV following bariatric surgery induced weight loss. Paradoxically, Cheng and colleagues (Cheng et al., 2013) demonstrated a reduction in $\mathrm{CD}_{144^{+}} \mathrm{EMV}$ (CD144 was part of our EMV multimarker panel that was unchanged by our LCD) while Stephanian and colleagues (2013) reported no change in $\mathrm{CD} 31^{+} 41^{-} \mathrm{EMV}$.

Of the various circulating MV populations, those of endothelial origin are typically the most difficult to enumerate. This may relate to the low abundance in the circulation of EMV above the noise of the instrument and/or to the low expression of endothelialspecific markers on the MV membrane. $\mathrm{CD} 31^{+} \mathrm{EMV}$ are the most widely reported in the literature, but the $\mathrm{CD} 31^{+}$EMV assay must include a platelet-specific marker to exclude PMV, as CD31 is also abundantly expressed on platelets. Unlike CD42b, sometimes employed in EMV research for this purpose, the expression of CD41 on platelets is relatively stable and not affected by platelet activation triggers, hence our definition of 
EMV as $\mathrm{CD} 31^{+} 41^{-}$events. A novel strategy that avoids the need to gate out PMV in a two colour assay is the monochrome multimarker assay proposed by Duval and colleagues (2010). This employs more than one endothelial-specific antibody, all tagged with the same fluorochrome to increase the signal of EMV bearing weakly expressed markers. Using this monochrome multimarker approach, Duval et al. reported a 20 fold difference between EMV of lupus patients and healthy controls. Although the use of single endothelial-specific markers is the ideal, this has not to date yielded clear EMV populations in our laboratory with the technology available. The lack of consensus on how to quantify EMV is a limitation in the field. There is scope for methodological research, possibly corroborating flow cytometric methods with ELISA-based MV assays or investigating other endothelial-specific markers such as thrombomodulin (Martin et al., 2014).

The effects of the LCD on protein markers of endothelial activation and acute phase inflammatory proteins are broadly in line with the results of a small number of other studies in this field (Davis et al., 2011; Keogh et al., 2008; Sharman \& Volek, 2004; Wycherley et al., 2009) with sE-selectin, sP-selectin, sTM, SAA and CRP lower at the end of the LCD phase. Previous studies have reported reductions in sTM (Porreca et al., 2004) and SAA (Yang et al., 2006) following a traditional hypocaloric diet leading to weight loss, though to our knowledge, this is the first study to demonstrate their reduction following a LCD intervention. Soluble P-selectin is present on both endothelial cells and platelets and the source of the LCD difference is unclear. Regardless, these changes are consistent with the cross talk that occurs between endothelial cells, platelets and 
leukocytes that occur at all stages of atherosclerotic disease (Siegel-Axel \& Gawaz, 2007). Contrary to other studies (Davis et al., 2011; Keogh et al., 2008; Sharman \& Volek, 2004; Wycherley et al., 2009), there was no difference in sICAM-1 during the LCD and ND phases. Crucially however, no marker measured was higher during the LCD phase. Differential responses of soluble selectins and cell adhesions molecules to diet and pharmacological intervention are common in the literature (Abe et al., 1998; Eschen et al., 2004; Hackman et al., 1996; Wycherley et al., 2009) with sVCAM-1 the least likely to be reduced. Hackman and colleagues (1996) speculated that the much of sICAM-1 and sVCAM-1 may arise not from the endothelium but from leukocytes and vascular smooth muscle cells, which do not always respond in the same way to treatment.

The mechanisms underpinning the reduction in EMV during the LCD phase are unclear. The reduction in EMV and other markers were accompanied by a modest reduction in body mass and waist circumference. Adipose tissue, particularly visceral adipose tissue is known to release pro-inflammatory cytokines such as tumour necrosis factor alpha $(\mathrm{TNF}-\alpha)$ that stimulate the release of EMV (Brown et al., 2011). Although not measured in this study, TNF- $\alpha$ is known to be reduced following weight loss (Dandona et al., 1998). These cytokines can also stimulate the production of the acute phase proteins CRP and SAA, with both proteins lower in the LCD phase of this study. A correlation was observed between the difference in body mass and the differences in CRP and sEselectin between diet phases, with a correlation also observed between the differences in waist circumference and mmEMV. Cheng and colleagues (Cheng et al., 2013) reported a considerable reduction in EMV one month following bariatric surgery coinciding with a 
$10 \%$ reduction in body mass, though there were no further reduction in EMV at 12 months, despite another $10 \%$ reduction in body mass. The time course for reductions in EMV may therefore not always mirror reductions in body mass. Hypertension is associated with elevated EMV (Chen et al., 2011), consistent with the mechanosensitive properties of endothelial cells. However, the small difference in diastolic blood pressure between dietary phases in this study was not correlated with the difference in EMV. Changes in macronutrient intake are other possibilities that could potentially influence EMV release, independent of weight loss. High glucose can induce oxidative stress in endothelial cells triggering EMV release (Jansen et al., 2013), with EMV elevated in diabetes (Leroyer et al., 2008). Although carbohydrate intake was reduced considerably during the LCD phase, fasting serum glucose was not different between phases however. It is not clear if a reduced carbohydrate intake can reduce endothelial stress in the absence of changes in fasting glucose. Further research is clearly needed to elucidate the mechanisms by which a LCD, leading to moderate weight loss, can lower EMV. In particular, it would be intriguing to examine the influence of a LCD on EMV and other endothelial biomarkers where body mass is maintained via increases in fat and protein intake.

The difference in EMV and other markers cannot be attributed to differences in blood lipids as no blood lipid or apolipoprotein variable was significantly different between the LCD and ND conditions. The absence of any absolute increase in fat, saturated fat and protein intake during the LCD phase may be relevant here. The effects on EMV of VLCD that result in pro-atherogenic changes in blood lipids have yet to be determined. 
As considerable biomarker reductions can occur in the absence of any change in blood lipids, these results also highlight the need to go beyond blood lipids when evaluating dietary interventions.

This study has a number of limitations. The study design did not involve a washout between trial phases with the mid-trial blood sample representing the end of the initial phase (ND or LCD phase) and the start of the next phase. This was in part due to the absence of data indicating the duration of a wash out necessary following the LCD phase to return biomarkers to pre-study concentrations but also a desire not to prolong the study beyond 48 weeks. Twenty-four weeks (duration of second phase) back on normal diets was likely to be sufficient however for a return to normal metabolic and endothelial profiles in those who were randomised to undertake the LCD phase first. Crucially, there was no evidence of carryover between phase 1 and phase 2 , with no significant differences in ND - LCD delta scores in those first randomised to the ND and those first randomised to the LCD. As a result, the effects of the LCD were determined simply by comparing participant values at the end of their LCD and ND phases. The results of this study do not generalise to all LCD. With a mean carbohydrate intake of $87 \mathrm{~g}^{\cdot \mathrm{day}^{-1}}$, representing $29 \%$ of daily energy needs, this LCD was not particularly extreme, though still reducing carbohydrate intake by $\sim 90 \mathrm{~g}^{\cdot \mathrm{day}^{-1}}{ }^{-}$More extreme ketogenic diets may have adverse effects on endothelial health, regardless of the magnitude of weight loss.

In conclusion, a diet that reduces carbohydrate intake by $\sim 90 \mathrm{~g}^{\cdot d a y^{-1}}$ so that carbohydrate accounts for $\sim 30 \%$ of total daily energy intake, results in modest reductions in body 
mass, waist circumference and blood pressure accompanied by reductions in $\mathrm{CD} 31^{+} \mathrm{CD} 41^{-} \mathrm{EMV}$ and multiple other endothelial inflammatory biomarkers. It does not increase any endothelial biomarker. Though the precise mechanisms involved are unclear from this study, the changes in endothelial markers can occur in the absence of any change in blood lipids. 


\section{Acknowledgments}

We are grateful to the volunteers who gave their time to take part in the study.

\section{Financial Support}

The study was funded by Technological Sector Research Strand I and Strand III grants to Waterford Institute of Technology

\section{Conflict of Interest}

None

\section{Authorship}

ALW, OOD, KSC and MH formulated the research question. $\mathrm{MH}$ and LD were responsible for the study design. ALW, DF, JPP and MDR were responsible for data collection and sample analysis. ALW and MH analysed the data. AW, MH, OOD and KSC prepared the manuscript. All authors approved the final manuscript version. 


\section{References}

Abe, Y., El-Masri, B., Kimball, K. T., Pownall, H., Reilly, C. F., Osmundsen, K., et al. 1998. Soluble cell adhesion molecules in hypertriglyceridemia and potential significance on monocyte adhesion. Arterioscler Thromb Vasc Biol, 18(5), 723 731.

Akers, J. C., Gonda, D., Kim, R., Carter, B. S., \& Chen, C. C. 2013. Biogenesis of extracellular vesicles (ev): Exosomes, microvesicles, retrovirus-like vesicles, and apoptotic bodies. J Neurooncol, 113(1), 1-11.

Andriantsitohaina, R., Gaceb, A., Vergori, L., \& Martinez, M. C. 2012. Microparticles as regulators of cardiovascular inflammation. Trends Cardiovasc Med, 22(4), 88-92.

Ayers, L., Nieuwland, R., Kohler, M., Kraenkel, N., Ferry, B., \& Leeson, P. 2015. Dynamic microvesicle release and clearance within the cardiovascular system: Triggers and mechanisms. Clin Sci (Lond), 129(11), 915-931.

Bilsborough, S. A., \& Crowe, T. C. 2003. Low-carbohydrate diets: What are the potential short- and long-term health implications? Asia Pac J Clin Nutr, 12(4), 396-404.

Brehm, B. J., Seeley, R. J., Daniels, S. R., \& D'Alessio, D. A. 2003. A randomized trial comparing a very low carbohydrate diet and a calorie-restricted low fat diet on body weight and cardiovascular risk factors in healthy women. J Clin Endocrinol Metab, 88(4), 1617-1623.

Brevetti, G., Schiano, V., \& Chiariello, M. 2006. Cellular adhesion molecules and peripheral arterial disease. Vasc Med, 11(1), 39-47.

Brown, M. D., Feairheller, D. L., Thakkar, S., Veerabhadrappa, P., \& Park, J. Y. 2011. Racial differences in tumor necrosis factor-alpha-induced endothelial microparticles and interleukin-6 production. Vasc Health Risk Manag, 7, 541-550.

Chen, Y., Feng, B., Li, X., Ni, Y., \& Luo, Y. 2011. Plasma endothelial microparticles and their correlation with the presence of hypertension and arterial stiffness in patients with type 2 diabetes. J Clin Hypertens (Greenwich), 14(7), 455-460.

Cheng, V., Kashyap, S. R., Schauer, P. R., Kirwan, J. P., \& McCrae, K. R. 2013. Restoration of glycemic control in patients with type 2 diabetes mellitus after bariatric surgery is associated with reduction in microparticles. Surg Obes Relat Dis, 9(2), 207-212.

Chironi, G. N., Boulanger, C. M., Simon, A., Dignat-George, F., Freyssinet, J. M., \& Tedgui, A. 2009. Endothelial microparticles in diseases. Cell Tissue Res, 335(1), 143-151.

Dandona, P., Weinstock, R., Thusu, K., Abdel-Rahman, E., Aljada, A., \& Wadden, T. 1998. Tumor necrosis factor-alpha in sera of obese patients: Fall with weight loss. J Clin Endocrinol Metab, 83(8), 2907-2910.

Davis, N. J., Crandall, J. P., Gajavelli, S., Berman, J. W., Tomuta, N., Wylie-Rosett, J., et al. 2011. Differential effects of low-carbohydrate and low-fat diets on inflammation and endothelial function in diabetes. J Diabetes Complications, 25(6), 371-376.

Duval, A., Helley, D., Capron, L., Youinou, P., Renaudineau, Y., Dubucquoi, S., et al. 2010. Endothelial dysfunction in systemic lupus patients with low disease activity: Evaluation by quantification and characterization of circulating 
endothelial microparticles, role of anti-endothelial cell antibodies. Rheumatology (Oxford), 49(6), 1049-1055.

Eschen, O., Christensen, J. H., De Caterina, R., \& Schmidt, E. B. 2004. Soluble adhesion molecules in healthy subjects: A dose-response study using n-3 fatty acids. Nutr Metab Cardiovasc Dis, 14(4), 180-185.

Foster, G. D., Wyatt, H. R., Hill, J. O., McGuckin, B. G., Brill, C., Mohammed, B. S., et al. 2003. A randomized trial of a low-carbohydrate diet for obesity. N Engl J Med, 348(21), 2082-2090.

Frigolet, M. E., Ramos Barragan, V. E., \& Tamez Gonzalez, M. 2011. Low-carbohydrate diets: A matter of love or hate. Ann Nutr Metab, 58(4), 320-334.

Gardner, C. D., Kiazand, A., Alhassan, S., Kim, S., Stafford, R. S., Balise, R. R., et al. 2007. Comparison of the atkins, zone, ornish, and learn diets for change in weight and related risk factors among overweight premenopausal women: The a to $\mathrm{z}$ weight loss study: A randomized trial. Jama, 297(9), 969-977.

Hackman, A., Abe, Y., Insull, W., Jr., Pownall, H., Smith, L., Dunn, K., et al. 1996. Levels of soluble cell adhesion molecules in patients with dyslipidemia. Circulation, 93(7), 1334-1338.

Jansen, F., Yang, X., Franklin, B. S., Hoelscher, M., Schmitz, T., Bedorf, J., et al. 2013. High glucose condition increases nadph oxidase activity in endothelial microparticles that promote vascular inflammation. Cardiovasc Res, 98(1), 94106.

Jimenez, J. J., Jy, W., Mauro, L. M., Soderland, C., Horstman, L. L., \& Ahn, Y. S. 2003. Endothelial cells release phenotypically and quantitatively distinct microparticles in activation and apoptosis. Thromb Res, 109(4), 175-180.

Keogh, J. B., Brinkworth, G. D., Noakes, M., Belobrajdic, D. P., Buckley, J. D., \& Clifton, P. M. 2008. Effects of weight loss from a very-low-carbohydrate diet on endothelial function and markers of cardiovascular disease risk in subjects with abdominal obesity. Am J Clin Nutr, 87(3), 567-576.

Lee, S. T., Chu, K., Jung, K. H., Kim, J. M., Moon, H. J., Bahn, J. J., et al. 2012. Circulating cd62e+ microparticles and cardiovascular outcomes. PLoS One, 7(4), e35713.

Leroyer, A. S., Tedgui, A., \& Boulanger, C. M. 2008. Microparticles and type 2 diabetes. Diabetes Metab, 34 Suppl 1, S27-32.

Martin, F. A., McLoughlin, A., Rochfort, K. D., Davenport, C., Murphy, R. P., \& Cummins, P. M. 2014. Regulation of thrombomodulin expression and release in human aortic endothelial cells by cyclic strain. PLoS One, 9(9), e108254.

Martin, F. A., Murphy, R. P., \& Cummins, P. M. 2013. Thrombomodulin and the vascular endothelium: Insights into functional, regulatory, and therapeutic aspects. Am J Physiol Heart Circ Physiol, 304(12), H1585-1597.

National Institutes of Health. 1998. Clinical guidelines on the identification, evaluation, and treatment of overweight and obesity in adults: Executive summary. Expert panel on the identification, evaluation, and treatment of overweight in adults. Am J Clin Nutr, 68(4), 899-917.

Nordmann, A. J., Nordmann, A., Briel, M., Keller, U., Yancy, W. S., Jr., Brehm, B. J., et al. 2006. Effects of low-carbohydrate vs low-fat diets on weight loss and 
cardiovascular risk factors: A meta-analysis of randomized controlled trials. Arch Intern Med, 166(3), 285-293.

Nozaki, T., Sugiyama, S., Koga, H., Sugamura, K., Ohba, K., Matsuzawa, Y., et al. 2009. Significance of a multiple biomarkers strategy including endothelial dysfunction to improve risk stratification for cardiovascular events in patients at high risk for coronary heart disease. J Am Coll Cardiol, 54(7), 601-608.

Porreca, E., Di Febbo, C., Fusco, L., Moretta, V., Di Nisio, M., \& Cuccurullo, F. 2004. Soluble thrombomodulin and vascular adhesion molecule-1 are associated to leptin plasma levels in obese women. Atherosclerosis, 172(1), 175-180.

Samaha, F. F., Iqbal, N., Seshadri, P., Chicano, K. L., Daily, D. A., McGrory, J., et al. 2003. A low-carbohydrate as compared with a low-fat diet in severe obesity. $\mathrm{N}$ Engl J Med, 348(21), 2074-2081.

Sharman, M. J., \& Volek, J. S. 2004. Weight loss leads to reductions in inflammatory biomarkers after a very-low-carbohydrate diet and a low-fat diet in overweight men. Clin Sci (Lond), 107(4), 365-369.

Siegel-Axel, D. I., \& Gawaz, M. 2007. Platelets and endothelial cells. Semin Thromb Hemost, 33(2), 128-135.

Stepanian, A., Bourguignat, L., Hennou, S., Coupaye, M., Hajage, D., Salomon, L., et al. 2013. Microparticle increase in severe obesity: Not related to metabolic syndrome and unchanged after massive weight loss. Obesity (Silver Spring), 21(11), 22362243.

Wekesa, A. L., Cross, K. S., O'Donovan, O., Dowdall, J. F., O'Brien, O., Doyle, M., et al. 2014. Predicting carotid artery disease and plaque instability from cell-derived microparticles. Eur J Vasc Endovasc Surg, 48(5), 489-495.

Wellek, S., \& Blettner, M. 2012. On the proper use of the crossover design in clinical trials: Part 18 of a series on evaluation of scientific publications. Dtsch Arztebl Int, 109(15), 276-281.

Wycherley, T. P., Brinkworth, G. D., Keogh, J. B., Noakes, M., Buckley, J. D., \& Clifton, P. M. 2009. Long-term effects of weight loss with a very low carbohydrate and low fat diet on vascular function in overweight and obese patients. J Intern Med, 267(5), 452-461.

Yancy, W. S., Jr., Olsen, M. K., Guyton, J. R., Bakst, R. P., \& Westman, E. C. 2004. A low-carbohydrate, ketogenic diet versus a low-fat diet to treat obesity and hyperlipidemia: A randomized, controlled trial. Ann Intern Med, 140(10), 769777.

Yang, R. Z., Lee, M. J., Hu, H., Pollin, T. I., Ryan, A. S., Nicklas, B. J., et al. 2006. Acute-phase serum amyloid A: An inflammatory adipokine and potential link between obesity and its metabolic complications. PLoS Med, 3(6), e287. 
Table 1 Daily energy, carbohydrate, protein, fat and saturated fat intake during the normal diet and low carbohydrate diet phases $(n=24)$

\section{Normal diet}

\section{Low carbohydrate diet}

Energy (kcal)

Carbohydrate (g)

Protein $(\mathrm{g})$

Fat (g)

Saturated fat (g)

$1626 \pm 86$
$179 \pm 11$
$71 \pm 3$
$70 \pm 5$
$27 \pm 2$

$179 \pm 11$

$70 \pm 5$

$27 \pm 2$

${ }^{*} p<0.05$ compared to Normal diet 
Table 2: Body composition and blood pressure following 24 weeks on a normal diet and 24 weeks on a low carbohydrate $\operatorname{diet}(n=24)$

\section{Normal diet Low carbohydrate diet}

Body mass (kg)

Body mass index $\left(\mathrm{kgm}^{-2}\right)$

Waist circumference $(\mathrm{cm})$

Systolic blood pressure $(\mathrm{mmHg})$

Diastolic blood pressure $(\mathrm{mmHg})$

${ }^{*} p<0.05$ compared to Normal diet

$\begin{array}{cc}73.8 \pm 1.8 & 70.1 \pm 1.8^{*} \\ 27.1 \pm 0.6 & 25.7 \pm 0.6 * \\ 85.8 \pm 0.5 & 82.3 \pm 0.6 * \\ 130 \pm 2 & 127 \pm 3 \\ 84 \pm 2 & 79 \pm 2 *\end{array}$

$73.8 \pm 1.8$

$79 \pm 2 *$ 
Table 3: Endothelial and inflammatory biomarkers following 24 weeks on a normal diet and 24 weeks on a low carbohydrate diet $(n=24)$

\begin{tabular}{|c|c|c|}
\hline & Normal diet & Low carbohydrate diet \\
\hline $\mathrm{CD} 1^{+} 41^{-} \mathrm{EMV}\left(\right.$ events $\left.^{-} \mu \mathrm{L}^{-1}\right)$ & $29.9 \pm 4.0$ & $20.9 \pm 3.0 *$ \\
\hline mmEMV (events $\mu \mathrm{L}^{-1}$ ) & $57.0 \pm 7.8$ & $43.9 \pm 6.8^{\dagger}$ \\
\hline sE-selectin $\left(\mathrm{ng}^{\prime} \mathrm{mL}^{-1}\right)$ & $13.1 \pm 1.4$ & $11.9 \pm 1.4^{*}$ \\
\hline $\mathrm{sP}-$ selectin $\left(\mathrm{ng}^{\cdot} \mathrm{mL}^{-1}\right)$ & $59.9 \pm 6.4$ & $52.3 \pm 5.7 *$ \\
\hline sICAM-1 (ng.mL $\left.{ }^{-1}\right)$ & $307 \pm 26$ & $294 \pm 37$ \\
\hline sVCAM-1 (ngmL $\left.L^{-1}\right)$ & $320 \pm 18$ & $320 \pm 14$ \\
\hline sThrombomodulin $\left(\mathrm{ngmL}^{-1}\right)$ & $3.62 \pm 0.23$ & $3.39 \pm 0.22 *$ \\
\hline sICAM-3 (ng:mL $\left.{ }^{-1}\right)$ & $1.25 \pm 0.18$ & $1.21 \pm 0.17^{\dagger}$ \\
\hline $\mathrm{CRP}\left(\mathrm{mg} \cdot \mathrm{L}^{-1}\right)$ & $1.49 \pm 0.31$ & $1.14 \pm 0.24 *$ \\
\hline SAA $\left(n g \cdot m L^{-1}\right)$ & $2556 \pm 570$ & $1546 \pm 415^{*}$ \\
\hline
\end{tabular}

${ }^{*} p<0.05$ compared to Normal diet. ${ }^{\dagger} p=0.08$ compared to Normal diet. Values are mean \pm SEM. EMV = endothelial microvesicles, $m m E M V=$ monochrome multimarker EMV and represents microvesicles positive for CD105, CD144 or CD146. Samples were stained with these three endothelial specific monochrome antibodies in order to increase EMV counts, sICAM-1 = soluble intercellular adhesion molecule 1, sVCAM-1 = soluble vascular cell adhesion molecule, $C R P=C$-reactive protein, $S A A=$ serum amyloid $A$ 
Table 4 Serum lipids and apolipoproteins following 24 weeks on a normal diet and 24 weeks on a low carbohydrate diet $(n=24)$

\begin{tabular}{lcc}
\hline & Normal diet & Low carbohydrate diet \\
\hline Apolipoprotein A1 $\left(\mathrm{mg} \cdot \mathrm{dL}^{-1}\right)$ & $138 \pm 3$ & $141 \pm 4$ \\
Apolipoprotein B $\left(\mathrm{mg}^{-1} \mathrm{dL}^{-1}\right)$ & $112 \pm 3$ & $110 \pm 4$ \\
Cholesterol $\left(\mathrm{mmol} \mathrm{L}^{-1}\right)$ & $5.5 \pm 0.1$ & $5.4 \pm 0.1$ \\
HDL-cholesterol $\left(\mathrm{mmol} \mathrm{L}^{-1}\right)$ & $1.67 \pm 0.07$ & $1.66 \pm 0.07$ \\
LDL-cholesterol $\left(\mathrm{mmol} \mathrm{L}^{-1}\right)$ & $3.3 \pm 0.1$ & $3.2 \pm 0.2$ \\
NEFA $\left(\mathrm{mmol} \cdot \mathrm{L}^{-1}\right)$ & $0.69 \pm 0.06$ & $0.61 \pm 0.03$ \\
Triglycerides $\left(\mathrm{mmol}^{-1}\right)$ & $0.92 \pm 0.06$ & $0.90 \pm 0.10$ \\
Glucose $\left(\mathrm{mmol} \cdot \mathrm{L}^{-1}\right)$ & $5.25 \pm 0.07$ & $5.26 \pm 0.12$ \\
\hline
\end{tabular}

Values are mean $\pm S E M$. HDL = high density lipoprotein; $L D L=$ low density lipoprotein; NEFA = Non-Esterified Fatty Acid 
Figure legends

Figure 1: Percentage of total daily energy intake obtained from carbohydrate, fat and protein when following a normal diet (ND) and a low carbohydrate diet (LCD) 


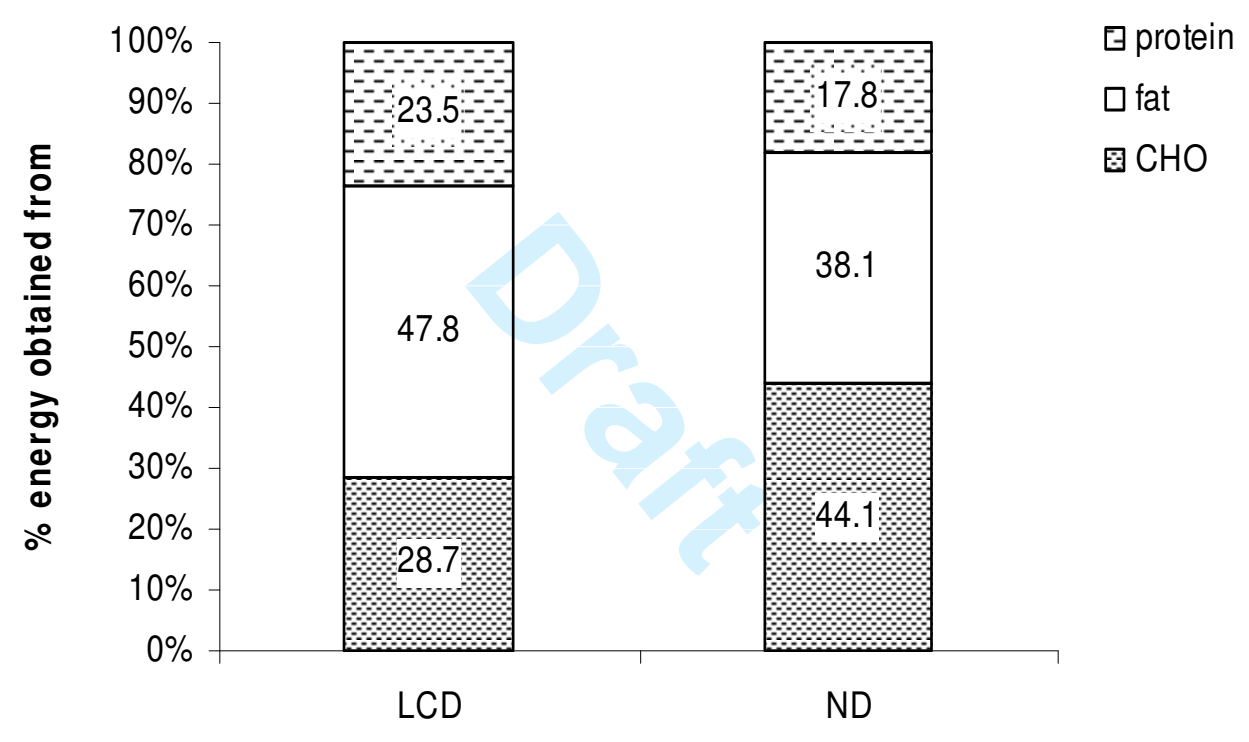

